\title{
The Origin and Nature of the Planck Constant
}

\author{
Nader Butto \\ Dania, Petah-Tikva, Israel \\ Email: nader.butto@gmail.com
}

How to cite this paper: Butto, N. (2021) The Origin and Nature of the Planck Constant. Journal of High Energy Physics, Gravitation and Cosmology, 7, 324-332. https://doi.org/10.4236/jhepgc.2021.71016

Received: November 5, 2020

Accepted: January 26, 2021

Published: January 29, 2021

Copyright $\odot 2021$ by author(s) and Scientific Research Publishing Inc. This work is licensed under the Creative Commons Attribution International License (CC BY 4.0).

http://creativecommons.org/licenses/by/4.0/

\begin{abstract}
Planck's constant $h$ is a fundamental physical constant defined in the realm of quantum theory and is determined only by physical measurement and cannot be calculated. To this day, physicists do not have a convincing explanation for why action in microcosm is quantized or why $h$ has a specific quantitative value. Here, a new theory is presented based on the idea that the elementary particles are vortices of a condensed superfluid vacuum. The vortex has a conserved angular momentum that can be calculated by applying hydrodynamic laws; in this way, the numerical value of Planck's constant can be obtained. Therefore, the Planck constant is not a fundamental constant but an observable parameter of the elementary particle as a vortex that has constant vorticity and conserved angular momentum. This theory may offer a unique and comprehensive understanding of Planck's constant and open a new perspective for a theory of everything.
\end{abstract}

\section{Keywords}

Planck's Constant, Angular Momentum, Compton Radius, Vorticity

\section{Introduction}

Max Planck's attempts to provide a theoretical explanation for the empirically discovered laws of blackbody radiation yielded Planck's constant $h$ that first appeared in physics theory in 1900 [1]. He proposed the quantum hypothesis stating that the energy of a harmonic oscillator with an oscillation frequency $v$ would quantize at an integral multiple of $h v$. Therefore, Planck's constant is the currently accepted quantum (smallest quantity) of energy possible within a photon and relates the energy in one quantum (photon) of electromagnetic radiation to the frequency of that radiation. The implication of discovery of $h$ was that the action of atoms is quantized and that $h$ represents the fundamental unit of action for discrete atomic-scale systems. It has become an integral component of modern atomic and subatomic physics and has profound importance in 
technology, understanding of reality, and understanding of life.

The estimated value of Planck's constant according to the 1998 CODATA was determined based on balancing electric and gravitational forces in a so-called watt balance [2]. In this scheme, the weight of a test mass is compared to the force generated by a coil, the electric power of which is accurately measured via the Josephson and quantum-Hall effects. The number chosen for the numerical value of the $h$ is such that, at the time of adopting the definition, one kilogram is equal to the mass of the international prototype currently used for the definition of mass, within the uncertainty of the combined best estimates of the value of the $h$ at that moment.

Another way to measure it is via the X-ray crystal density (XRCD) method [3]. This method measures the Avogadro constant $N_{\mathrm{A}}$ to establish a mass scale by counting the number of atoms in a silicon single crystal sphere using the $\mathrm{XRCD}$ approach, i.e., probing the regular arrangement of atoms in a perfect lattice, and then multiplying it by the known mass of a silicon atom (the ${ }^{28} \mathrm{Si}$ isotope) [4].

The CODATA Recommended Values of the Fundamental Physical Constants used these measurement results and the measurement results of the abovementioned watt balance method as a basis to determine Planck's constant, which is $h$ $=6.6260693 \times 10^{-34} \mathrm{~J} \mathrm{~s}[5]$.

Progress is made every year in measuring Planck's constant; however, little progress has been made in understanding its nature. Planck's constant is thought to be a fundamental physical constant defined in the realm of quantum theory; however, thus far, physicists do not have a convincing explanation for why action in the microcosm is quantized or why $h$ has a specific quantitative value [6].

In previous articles, the nature and the origin of the fine structure constant [7], the gravitational constant $G$ [8], magnetic constant $\mu_{0}$ [9] and electric permittivity [10] were described.

In this paper, we provide a new theory to describe the origin of Planck's constant and to reveal the constant's intrinsic nature. The starting point is the superfluid nature of the vacuum, which explains the vortex nature of the elementary particles. Thereafter, applying the classical laws of hydrodynamics to the vortex to calculate the vorticity and angular momentum of the vortex, an analytical formulation is presented to obtain the numerical value of the constant.

\section{Superfluid Vacuum}

Although the theory of quantum mechanics is not predicted based upon any property of space, the idea of space is frequently used to justify mathematical procedures and to imply the amounts of detailed space properties such as the speed of light in a vacuum governed by the vacuum permeability and permittivity. During the early years of quantum mechanics, Paul Dirac theorized that vacuum was actually filled with particles in negative energy states [11], therefore 
giving rise to the concept of the "physical vacuum," which is not empty at all. In quantum electrodynamics, the vacuum is a state with no matter particles and photons but with vacuum fluctuations and with a finite energy called the vacuum energy. The vacuum is defined as the state with the lowest possible energy and a superfluid behavior. The superfluidity of the vacuum is the basis for Maxwell's equations, special relativity, and general relativity.

The classical behavior of the electromagnetic field is described by Maxwell's equations, which predict that the speed of light $c$ in which electromagnetic waves (such as light) propagate through the vacuum, is related to the electric constant $\varepsilon_{0}$ and magnetic constant $\mu_{0}$. Special relativity is derived from Maxwell's equations. Einstein clearly realized that both special and general relativity were based on fluid dynamical models [12].

Nonetheless, the microscopic structure of the vacuum is currently largely unknown according to quantum field theory. Even in the absence of real particles, the vacuum is always filled by pairs of created and annihilated virtual particles, and it is predicted that these invisible particles could materialize for a short time and exert a measureable force. Therefore, the physical vacuum is assumed to be a non-trivial medium, not empty but rather filled with quantum mechanical zero-point energy and characterized as behaving like a frictionless fluid with extremely low viscosity, in which one can associate a certain energy and density with extremely high thermal conductivity. Therefore, the vacuum energy has real physically observable consequences, and its properties can be observed as having real physical effects [13] [14].

The vacuum extends everywhere, has no size, shape, center, direction, time, or extent, and is immovable. Therefore, the vacuum density is generally viewed as a fundamental property of the cosmos, its magnitude should not depend on whether we choose subatomic, astronomical, or cosmological methods to assess its value.

The vacuum density value depends primarily on general relativity, and has been determined using astronomical observations of the curvature of space-time and the expansion of the universe. The expansion of the universe has been studied using several different methods; however, the Wilkinson Microwave Anisotropy Probe mission represents a major step toward precision in calculating the Hubble constant and the vacuum density [15].

The most recent result [16], indicates that the value of the Hubble constant is $H_{0}=71.9+2.4-3.0 \mathrm{~km} / \mathrm{s} / \mathrm{Mpc}=2.33 \times 10^{-18} \mathrm{~s}^{-1}$, where the number of $\mathrm{km}$ in an $\mathrm{Mpc}$ is $3.09 \times 10^{19}$. Considering that the inertial mass of the Observable Universe is $M_{\mathrm{U}}=c^{3} 2 H_{0} G=0.8720532288 \times 10^{56} \mathrm{~kg}$ and the volume of the universe is $V_{\mathrm{U}}=4 / 3 \pi R_{\mathrm{U}}^{3}=4 / 3 \pi\left(c / H_{0}\right)^{3}=8.9364367479 \times 10^{81} \mathrm{~m}^{3}$, the cosmological density is calculated to be $M_{\mathrm{U}} / V_{\mathrm{U}}=9.75839983 \times 10^{-27} \mathrm{~kg} / \mathrm{m}^{3}$.

\section{Elementary Particles as Vortices}

The angular momentum (spin) of an electron indicates that there is an internal 
rotation that confers its rest mass. It has become obvious that not only an internal oscillation but also some type of internal motion at the speed of light is present. Therefore, the seemingly empty space that surrounds electrons is composed of "virtual particles" and electrons are inseparable from the clouds of virtual particles that surround them.

Quantum mechanics predicts that an electron is composed a cloud of probabilities. Although the precise measuring of the form of this cloud is beyond the capability of modern methods, the current model predicts that electrons are slightly aspheric, with a distortion characterized by the electric dipole moment. However, no experiment has ever detected this deviation [17]. Therefore, we propose that elementary particles, such as quarks and electrons, are irrotational circular vortices of frictionless superfluid space with concentric streamlines generated from the primordial vacuum during the Big Bang. The rate of rotation of the fluid is greatest at the center and decreases progressively with distance from the center until there is no gradient pressure on the boundaries of the vortex where the flow is laminar and the friction is zero. In such a case, the absence of friction would make it impossible to create or destroy the vortex motion. If the negative suction point-volume in the center of the vortex does not have sufficient energy to drag the virtual photons to the speed of light, a stable situation cannot occur [18].

In previous article [19], the electron properties have been accurately described using classical laws of hydrodynamics and describing the electron as a vortex.

\section{Hydrodynamics of the Vortex}

In hydrodynamics, the force $F$ that moves the vortex is directly related to the pressure that creates the vortex, known as the dynamic pressure $P_{d}$ and the area $A$ :

$$
F=P_{d} A .
$$

The dynamic pressure $\left(P_{d}\right)$ representing the fluid kinetic energy is expressed as

$$
P_{d}=1 / 2 \rho v^{2},
$$

where $\rho$ is the density of the fluid and $v=c$ is the velocity of the fluid.

Therefore, the internal force of the vortex is

$$
F=1 / 2 \rho c^{2} A .
$$

The area of the vortex is approximately a circle, and its radius when the vortex is extended will cause the vortex radius to double in size. Therefore, $A=2 \pi r^{2}$ and

$$
F=\rho c^{2} \pi r^{2} .
$$

If we multiply and divide the right hand side of the equation by time $t$ we obtain

$$
F=\rho c t \pi r^{2} c / t .
$$

The quantity $c t$ is equivalent to the distance $L, L \pi r^{2}$ is equivalent to the vo- 
lume $Q, \rho Q$ is equivalent to the mass $m$, and $1 / t$ is equivalent to the frequency $f$; therefore,

$$
F=m c f .
$$

The energy of the rotating electron around its axis is $E=$ force $\times$ distance. The distance that an electron rotates in one cycle is $2 \pi r$, therefore,

$$
E=2 \pi r c m f \text {. }
$$

This energy is the energy assigned to virtual particles. In this case, the frequency indicates the number of passages of one electromagnetic wave within one second of time. Planck's constant is the energy found within one wave.

In hydrodynamics, the velocity of a fluid element instantaneously passing through a given point in space in a vortex with a radius $r$ would be constant in time; therefore, the circulation or the vorticity is $\Gamma_{\mathrm{e}}=2 \pi r_{\mathrm{e}} c$. This is a fundamental constant for every vortex, as long as it exists in time and space, and vanishes only upon the destruction of the vortex. The quantity $\Gamma_{e} m_{e}$ is an angular momentum; therefore, $2 \pi r_{\mathrm{e}} c m_{\mathrm{e}}$ is a constant.

If we consider the Compton wavelength, $2.4263102367(11) \times 10^{-12} \mathrm{~m}$, to be one rotation of a vortex that has a core circumference of $2 \pi r$, the Compton radius is $2.4263102367(11) \times 10^{-12} / 2 \pi=3.86 \times 10^{-13} \mathrm{~m}$.

If the radius of the core of the vortex is $3.86 \times 10^{-13} \mathrm{~m}, c=2.99792458 \times 10^{8}$ $\mathrm{m} \cdot \mathrm{s}^{-1}$, and $m$ is the rest mass of an electron $m_{\mathrm{o}}=9.10938356 \times 10^{-31} \mathrm{~kg}$, the angular momentum is $2 \pi \mathrm{rcm}=6.61997943364 \times 10^{-34} \mathrm{~kg} \cdot \mathrm{m}^{2} \cdot \mathrm{s}^{-1}$, which is within the range of the discrepancies in the experimental values.

How is $2 \pi \mathrm{rcm}$ related to the Planck constant?

According to Planck theory, for photons of a frequency $f$, energy is given by

$$
E=h f \text {, }
$$

and the electron's rest mass energy $E_{0}$ can be represented by the following formula:

$$
E_{0}=m c^{2} .
$$

Therefore,

$$
h=m c^{2} / f .
$$

If the frequency $f=1 / t$, then

$$
h=m c^{2} t .
$$

In a vortex, the time necessary to complete one revolution is

$$
t=2 \pi r_{\mathrm{e}} / c .
$$

If we substitute the value of $t$ in Equation (12) into Equation (11), we obtain

$$
h=2 \pi r_{\mathrm{e}} \mathrm{cm} \text {. }
$$

If $\Gamma_{\mathrm{e}}=2 \pi r_{\mathrm{e}} \mathcal{C}, h=\Gamma_{\mathrm{e}} m_{\mathrm{e}}$, and $\Gamma_{\mathrm{e}}=h / m_{\mathrm{e}}=7.274$, then $r_{\mathrm{e}}=\Gamma_{\mathrm{e}} / 2 \pi c=3.86 \times 10^{-13} \mathrm{~m}$. This is the value of the Compton radius.

Examining the mathematical equations by dimensional analysis gives $M L^{2} / T$, which is the dimension of action, i.e., the energy multiplied by time; therefore, it 
is natural to think of $h$ in terms of action principles.

Therefore,

$$
E=2 \pi r_{e} c m f=h f \text {. }
$$

\section{Discussion}

The Planck constant and the speed of light are the two fundamental constants that rule the universe [20]. The speed of light is related to the strength of gravity, and Planck's constant relates energy to the frequency of a particle of light. All other constants, such as the charge or mass of an electron or the strength of the nuclear forces, can be described in relation to these two "dimensional" constants.

Planck's $h$ is very small and only comes into play at the "quantum" scale of very small things.

The effect of fixing the numerical value of the Planck constant is a definition of the unit $\mathrm{kg} \cdot \mathrm{m}^{2} \cdot \mathrm{s}^{-1}$ (the unit of the physical quantity called action). Its value has been determined experimentally using the XRCD and watt balance approaches. However, there are significant discrepancies between the available experimental values for the Planck constant [21].

Most recently in 2015, researchers from National Institute of Standards and Technology (NIST), USA) researchers reported a single value (NIST-15) for $h$ with an uncertainty of 5.6 parts in $10^{8}$ based on all the data obtained using their current watt balance [22]. Furthermore, also in 2015, the International Avogadro Collaboration (IAC) reported a new value (IAC-15) with an uncertainty of 2.0 parts in $10^{8}$ on the basis of the XRCD method; this value also fulfilled the condition of the second quantitative requirements of the Consultative Committee for Mass and Related Quantities for determinations of the Planck constant [23]. Progress is made every year in measuring Planck's constant; however, little progress has been made in understanding its nature.

We present a new perspective of an old idea that the electron is a vortex of superfluid vacuum. Superfluid vacuum theory proposes the mass generation mechanism, which may replace or supplement the electroweak Higgs mechanism. It has been shown that the masses of the elementary particles could emerge as the result of interactions with the superfluid vacuum, similar to the gap generation mechanism in superconductors [24] [25]. The super fluidity of the vacuum is the basis for Maxwell's equations. In deriving these equations, Maxwell made certain assumptions about the nature of the medium that carried electricity, magnetism, and light. The primary assumption used by Maxwell was that the underlying medium could be described using the perfect fluid vortex theory developed by Helmholtz. Therefore, we propose that the electron is an irrotational circular vortex of frictionless superfluid space with concentric streamlines and that is applies hydrodynamics to express the angular momentum of the vortex to connect it to Planck's constant.

From the Planck-Einstein equation we obtain $h=2 \pi r_{\mathrm{e}} \mathrm{cm}$, and from quantum 
mechanics the standard Compton wavelength, $\lambda$, of a particle is given by $\lambda=$ $h / m c$ and $h=\lambda \mathrm{cm}$. This indicates that $\lambda=2 \pi r_{\mathrm{e}}$; therefore, the Compton wavelength is the same as the electron core circumference. We obtained the Compton radius (which is different from the classical radius) and calculated the angular momentum of the core vortex, which gave the same value of Planck's constant. This is the first time, to our knowledge, that the Planck constant has been derived from an analytical formula based on the proposed theory, which explains the hydrodynamic mechanism of the angular constant as the origin of its quantitative value and provides a precise value of the Planck constant that can be expressed with a coherent set of units according to the International System of Units (SI units). The effect of fixing the numerical value of the Planck constant is a definition of the unit $\mathrm{kg} \cdot \mathrm{m}^{2} \cdot \mathrm{s}^{-1}$.

\section{Conclusions}

Planck's constant is an expression of the angular momentum of a frictionless vortex elementary particle composed of the condensed vacuum and generated in the Big Bang from massless virtual photons that acquire mass when moving in the vortex at the speed of light, as described by Higgs theory. The circulation in the vortex is constant, and the angular momentum of the vortex is conserved. By taking the Compton wavelength to be the circumference of the core vortex, we calculated the Compton wavelength and the angular momentum of the vortex to obtain the value of the Planck constant.

We conclude that the Planck constant is not a fundamental constant but an observable parameter of the elementary particle as a vortex, which expresses the circulation conserved momentum of the vortex. This theory may offer a unique and deeper understanding of Planck's constant and change the definitions of units to establish practical realizations by ever-increasingly accurate experiments.

\section{Acknowledgments}

The author would like to thank Enago (https://www.enago.com/) for the English language and peer reviewers review.

\section{Conflicts of Interest}

The author declares no conflicts of interest regarding the publication of this paper.

\section{References}

[1] Plank, M. (1900) Zur theorie des gesetzes der energieverteilung im normalspektrum. Verhandlungen der Deutschen Physikalischen Gesellschaft, 2, 237.

[2] Steiner, R., Williams, E.R., Liu, R. and Newell, D.B. (2007) Uncertainty Improvements of the NIST Electronic Kilogram. IEEE Transactions on Instrumentation and Measurement, 56, 592-596. https://doi.org/10.1109/TIM.2007.890590

[3] Massa, E. and Nicolaus, A. (2011) Special Issue International Determination of the Avogadro Constant. Metrologia, 48, S1-119. https://doi.org/10.1088/0026-1394/48/2/E01 
[4] Bettin, H., et al. (2013) Accurate Measurements of the Avogadro and Planck Constants by Counting Silicon Atoms. Annalen der Physik, 525, 680-687. https://doi.org/10.1002/andp.201300038

[5] Mohr, P.J. and Taylor, B.N. (2005) CODATA Recommended Values of the Fundamental Physical Constants: 2002. Reviews of Modern Physics, 77, 1.

https://doi.org/10.6028/NIST.SP.959e2005

[6] Peacock, K.A. (2008) The Quantum Revolution-A Historical Perspective. Greenwood Press, Westport and London.

[7] Butto, N. (2020) A New Theory on the Origin and Nature of the Fine Structure Constant. Journal of High Energy Physics, Gravitation and Cosmology, 6, 579-589. https://doi.org/10.4236/jhepgc.2020.64039

[8] Butto, N. (2020) New Mechanism and Analytical Formula for Understanding the Gravity Constant G. Journal of High Energy Physics, Gravitation and Cosmology, 6, 357-367. https://doi.org/10.4236/jhepgc.2020.63029

[9] Butto, N. (2020) The Essence and Origin of the Magnetic Constant. Journal of High Energy Physics, Gravitation and Cosmology, 6, 662-669. https://doi.org/10.4236/jhepgc.2020.64045

[10] Butto, N. (2021) Revealing the Essence of Electric Permittivity Constant. Journal of High Energy Physics, Gravitation and Cosmology, 7, 210-217.

[11] Dirac, P.A. (1930) A Theory of Electrons and Protons. Proceedings of the Royal Society of London A: Mathematical, Physical and Engineering Sciences, 126, 360-365. https://doi.org/10.1098/rspa.1930.0013

[12] Condon, E.U. and Odishaw, H. (1958) Handbook of Physics, Section 29. McgrawHill, New York, 2-50.

[13] Rauscher, E.A. (1968) Electron Interactions and Quantum Plasma Physics. Journal of Plasma Physics, 2, 517-541. https://doi.org/10.1017/S0022377800004013

[14] Rauscher, E.A. (2004) Dynamic Plasma Excitation Modes of Propagation in the Ionosphere. PA Press, The University of Columbus, 277.

[15] Peebles, P.J.E. and Ratra, B. (2003) The Cosmological Constant and Dark Energy. Reviews of Modern Physics, 75, 559-606.

https://doi.org/10.1103/RevModPhys.75.559

[16] Bonvin, V., et al. (2017) H0LiCOW V. New COSMOGRAIL Time Delays of HE 0435-1223: H0 to 3.8\% Precision from Strong Lensing in a Flat $\Lambda$ CDM Model. Monthly Notices of the Royal Astronomical Society, 465, 4914-4930. https://doi.org/10.1093/mnras/stw3006

[17] Hudson, J.J., et al. (2011) Improved Measurement of the Shape of the Electron. Nature, 473, 493-496. https://doi.org/10.1038/nature10104

[18] Butto, N. (2020) Electron Shape and Structure: A New Vortex Theory. Journal of High Energy Physics, Gravitation and Cosmology, 6, 340-352. https://doi.org/10.4236/jhepgc.2020.63027

[19] Ball, P. (2007) Two Constants to Rule Us All. Nature. https://doi.org/10.1038/news.2007.389

[20] Mohr, P.J., Taylor, B.N. and Newell, D.B. (2012) CODATA Recommended Values of the Fundamental Physical Constants: 2010. Journal of Physical and Chemical Reference Data, 41, Article ID: 043109. https://doi.org/10.1103/RevModPhys.84.1527

[21] Schlamminger, S., et al. (2015) A Summary of the Planck Constant Measurements Using a Watt Balance with a Superconducting Solenoid at NIST. Metrologia, 52, 
L5-L8. https://doi.org/10.1088/0026-1394/52/2/L5

[22] Azuma, Y., et al. (2015) Improved Measurement Results for the Avogadro Constant Using a 28Si-Enriched Crystal. Metrologia, 52, 360-375.

https://doi.org/10.1088/0026-1394/52/2/360

[23] Zloshchastiev, K.G. (2011) Spontaneous Symmetry Breaking and Mass Generation as Built-In Phenomena in Logarithmic Nonlinear Quantum Theory. Acta Physica Polonica B, 42, 261-292.

[24] Avdeenkov, A.V. and Zloshchastiev, K.G. (2011) Quantum Bose Liquids with Logarithmic Nonlinearity: Self-Sustainability and Emergence of Spatial Extent. Journal of Physics B: Atomic, Molecular and Optical Physics, 44, Article ID: 195303. https://doi.org/10.1088/0953-4075/44/19/195303

[25] Maxwell, J. (1873) A Treatise on Electricity and Magnetism. Sections 822 and 823. 